\title{
FLT3-ITD Negative Refractory/Relapsed or Geratic AML Patients Responded Well to Salvage Treatment with Low Dose Cytarabine and Sorafenib
}

\author{
Xu Jianhui ${ }^{1}$, Yao Qianqian ${ }^{2}$, Zhong Yuxia ${ }^{1}$, He Yingzhi ${ }^{1}$, Du Jingwen ${ }^{1}$, Liu Minhong ${ }^{1}$, \\ Huang Yuxian ${ }^{1}$, Li Yuhua ${ }^{1}$, Wu Bingyi ${ }^{2, ~ * ~}$ \\ ${ }^{1}$ Hematology Department, Zhujiang Hospital, Southern Medical University, Guangzhou, China \\ ${ }^{2}$ Hematology Department, Shunde Hospital of Sourthern Medical University, Foshan, China
}

Email address:

wubingyi@aliyun.com (Wu Bingyi)

${ }^{*}$ Corresponding author

To cite this article:

Xu Jianhui, Yao Qianqian, Zhong Yuxia, He Yingzhi, Du Jingwen, Liu Minhong, Huang Yuxian, Li Yuhua, Wu Bingyi. FLT3-ITD Negative Refractory/Relapsed or Geratic AML Patients Responded Well to Salvage Treatment with Low Dose Cytarabine and Sorafenib. International Journal of Clinical and Experimental Medical Sciences. Vol. 5, No. 6, 2019, pp. 92-97. doi: 10.11648/j.ijcems.20190506.11

Received: October 23, 2019; Accepted: November 23, 2019; Published: December 2, 2019

\begin{abstract}
There are great needs to explore more efficient and low-cytotoxic treatment for refractory/relapsed (R/R) or old acute myeloid leukemia (AML) patients without FLT3-ITD mutation. We observed the procedures and outcomes of 13 such patients subsequently treated by sorafenib in our departments. Five of them used low-dose cytorabine concomitantly and 8 patients took sorafenib alone as induction therapy. Five patients achieved complete remission (CR) and the needed time ranged from 31 to 100 days. Four patients achieved CR by concomitantly using low-dose cytorabine and sorafenib, but only one patient achieved CR by taking sorafenib alone. The difference of CR induction rates between the two groups was significant. Sorafenib was then prescribed as the maintenance treatment to these AML patients achieving CR until adverse event happening or stem cell transplantation received. Only one patient developed adverse event of grade 3 during the maintenance with sorafenib and it was relieved by withdrawal of the drug. The event free survival with sorafenib ranged from 2 to 20 months. The median survival time of these CR patients was 520 days since the beginning of taking sorafenib. The median survival time of those not achieved CR with sorafenib was 344 days. But we found no significant differences of survival time between those achieving and not achieving CR. Our results of the observation suggested a group of R/R or geratic AML patients unfit for intensive chemotherapy induction and without FLT3-ITD mutation may well respond to single sorafenib treatment. The combination of low-dose cytarabine with sorafenib improved the response rate comparing with using sorafenib alone. This treatment is safe and the survival time is acceptable for such formidable patients.
\end{abstract}

Keywords: Sorafenib, Cytorabine, Refractory or Relapsed Acute Myeloid Leukemia, Old Acute Myeloid Leukemia, FLT3-ITD Mutation, Clinical Observation

\section{Introduction}

There are great unmet needs to explore more efficient and low-cytotoxic treatment for refractory or relapsed acute myeloid leukemia (R/R AML) patients. The first reason is that a minor part of AML patients can't achieve complete remission (CR) with presently recommended induction chemotherapies. The second reason is although there are more than $60 \%$ of AML patients (not acute promyelocytic leukemia) can achieve temporary CR in 3 months [1], more than half of these patients would have disease recurrences in the first year [2]. And the third reason is that the present strategies to treat the $\mathrm{R} / \mathrm{R}$ AML patients, for example salvage chemotherapies, have still limited long term benefits and considerable side effects [3-5].

As to the elderly patients with AML, to find the new therapy is more needed. First, the prognosis of this group was rather inferior to younger patients [6]. Second, considerable elderly patients were unfit for intensive induction chemotherapy based on age, performance status (PS) and 
comorbidities with high risk of mortality in unfit patients [7]. Third, the adverse cytogenetics were more frequent in the elderly patiets and related with poor response to treatment as well as poor grognosis [8]. Forth, the low-intensity approaches such as low-dose cytorabine usually used in unfit patients could only achieve a low CR rate and no survival benefits were seen among patients who failed to achieve CR [9].

Sorafenib treatment, was reported to achieve better relapse-free survival and overall survival in less than 60 years old AML patients with FLT3-ITD mutation [10]. It was also reported to lead to an overall composite response rate of $46 \%$ with combinaton of azacitidine in $\mathrm{R} / \mathrm{R}$ AML including old patients with FLT3-ITD mutation [11]. Given sorafenib is a inhibitor to multi-targets tyrosine kinases besides FLT3, we hypothesized that it can also be useful with/without lowintense chemotherapy in $\mathrm{R} / \mathrm{R}$ AML or geratic AML patients without FLT3-ITD mutation. To see if it benefits this formidable group of patients, we made a retrospective observation of such patients in our center and report here.

\section{Methods}

\subsection{Patients and Data Collection}

Thirteen $\mathrm{R} / \mathrm{R}$ or elderly $\mathrm{AML}$ patients were sequentially treated in our center from May 2015 to December 2018. All of the patients signed to agree with the use of sorafenib as a single treatment or in combination with low-dose cytorabine on their AML. Before sorafenib useage, diagnosis was referred to the category criterion of WHO 2008. Patients' bone marrow was reserved for karyotypes and phenotypes analysis. Patients' bone marrow samples were tested for FLT3-ITD, NPM1, CEBPA, RUNX1, ASXL1 and TP53 gene mutations as previously described [12]. They were divided into three prognostic risk groups as reported [13].

Then patients were prescribed sorafenib (Nexavar ${ }^{\circledR}$ ) of 0.4 gram twice a day and some of the patients concomitantly used 10 to 14 days of low-dose cytorabine $\left(25 \mathrm{mg} / \mathrm{M}^{2}\right.$ subcutanous injection twice a day) for induction. Surveillance was scheduled to take bone marrow aspirate every one to two weeks in the first month and then every month later. Count of blood cell was undertaken every week since sorafenib administration. The prescription of sorafenib would go to cease if the bone marrow blast cell proportions increased in the latter two to three weeks comparing with before taking sorafenib. During the induction period, supportive cares were given with standard. Red blood cell transfusion was given at the threshold of no more than $6 \mathrm{~g} / \mathrm{dl}$ for hemoglobin or in the case of anemia symptoms deteriorating. Platelets are for patients with less than $20,000 / \mathrm{mcl}$ or with any signs of bleeding. Voriconazole or posaconazole were given in patients with neutropenia to prevent invasive fungal infections. G-CSF was subcutenously administered as long as neutrophils were less than $1000 / \mathrm{mcl}$. Salvage chemotherapy or allo-genetic hematopoietic cell transplantation would follow for those failed to chieve CR.
The patients achieving CR were also encouraged to receive allo-genetic hematopoietic cell transplantation according to their age and performance status. If transplantation was not possible, sorafenib would be given as a maintenance therapy until unendurable adverse event happening. All patients were followed up for their blood counts every month, or had their bone marrow tested once having blood cell abnormal.

\subsection{Response Assessment}

The standard of responses was defined as reported [16]. The complete remission (CR) fulfills the conditions of bone marrow blasts of less than $5 \%$ in an aspirate with spicules, transfusion independence with absolute neutrophils more than $1000 / \mathrm{mcl}$, platelets no less than $100,000 / \mathrm{mcl}$, and no residual evidence of extramedullary disease. The complete remission with incomplete blood count recovery (CRi) refers to the standard fulfill the complete remission except for the count of neutrophils or platelets. Partial remission (PR) refers to the decrease of at least $50 \%$ in the percentage of blasts to $5 \%$ to $25 \%$ in the bone marrow aspirate and the normalization of blood counts, as noted above.

\subsection{Side Effects Observation}

Patients were notified to report any signs of cardiac ischemia, such as chest/jaw/left arm pain, severe abdominal/stomach pain, and vomit, loss of balance, seizures, fainting, tachycardia, et al. Dermal lesions were also observed, such as blisters, redness, swelling and pain. Bleeding problems were notified, such as easy bruising or bleeding, tongue/mouth sores or pain, tingling or swollen of extremities. Signs of liver problems, such as stomach/abdominal pain, persistent nausea/vomiting, yellowing eyes/skin, dark urine, were observed. Except for counts of bone marrow blast cells and blood cells, electrolytes and liver or kidney functional biological parameters were monitored every other weeks. Side effect grade was named according to common terminology criteria for adverse events v 4.0 by US National Institutes of Health.

\subsection{Definitions}

Patients with persistent disease after two cycle of induction (either the standard ' $3+7$ ' regimen or in old patient the lowintense subcutaneous cytarabine included regimen) were regarded as having primary refractory disease. And relapse was defined as reappearance of leukemia in a patient with a previous documented $\mathrm{CR}$, including patients who underwent allo-stem-cell transplantation. The elderly patients unfit for intensive chemotherapy were difined by the age more than 75 or those aged $66-75$ with PS $\geq 2$ or those with obvious adverse cytogenetics and comorbidities [14].

\subsection{Statistical Analysis}

SAS6.0 (SAS Institute Inc., Cary, NC, USA) was used to make statistical analysis. The $\mathrm{CR}$ rate difference between those receiving sorafenib combining cytarabine and those using sorafenib alone was analyzed with the Chi-square test 
and the Fisher`s exact test.

\section{Results}

\subsection{Patients' Characteristics}

Seven from the 13 patients were female and the ages at onset ranged from 19 to 82 years old. The patients' diagnosis and former treatment were detailed in Table 1. There are 5 intermediate, 6 adverse and 2 favorable risk patients included. Ten patients were refractory or relapsed AML patients before taking sorafenib. Three old patients were unfit for convenient induction chemotherapy at onset. One of them had serious pneumonia and liver cirrhosis. One other had mitral regurgitation and atrial fibrillation at the time of diagnosis. And the third one was more than 80 years old and refused to receive chemotherapy. Four patients experienced relapses before taking sorafenib and their intervals between relapse and CR ranged from 1 to 18 months. Two patients had undergone stem cell transplantation but failed salvage chemotherapies. All patients were tested to be FLT3-ITD mutation negative.

Table 1. Patients' general status and chemotherapies used before sorafinib.

\begin{tabular}{|c|c|c|c|c|c|c|}
\hline Patient & Gender & $\begin{array}{l}\text { Onset } \\
\text { age }\end{array}$ & Diagnosis and Risk status & Previous treatments & $\begin{array}{l}\text { Treatment } \\
\text { response }\end{array}$ & $\begin{array}{l}\text { Relapse } \\
\text { Interval }\end{array}$ \\
\hline NO. 1 & female & 49 & $\begin{array}{l}\text { AML with maturation; adverse group with } \\
\text { normal karyotype but ASXL1 mutation }\end{array}$ & $\begin{array}{l}\text { Etoposide+ }+ \text { pirarubicin }+ \text { cytarabine Fludarabine+ } \\
\text { high-dose cytarabine }\end{array}$ & Refractory & \\
\hline NO. 2 & female & 52 & $\begin{array}{l}\text { AML with maturation; adverse group with } \\
\text { normal karyotype but ASXL1 mutation }\end{array}$ & Idarubicin + cytarabine for 2 rounds & Refractory & \\
\hline NO. 3 & female & 24 & $\begin{array}{l}\text { AML without maturation; favorable group } \\
\text { with normal karyotype and biallelic mutated } \\
\text { CEBPA }\end{array}$ & $\begin{array}{l}\text { Idarubicin }+ \text { cytarabine } \\
\text { Etoposide }+ \text { mitoxantrone }+ \text { cytarabine }\end{array}$ & $\begin{array}{l}\text { Relapsed } \\
\text { Refractory }\end{array}$ & 3 months \\
\hline NO. 4 & male & 68 & $\begin{array}{l}\text { AML with inv ( } 3 \text { ) (q22; 26); adverse group } \\
\text { with unfavorable karyotype }\end{array}$ & $\begin{array}{l}\text { Homoharringtonine+cytarabine } \\
\text { Pirarubicin+cytarabine Idarubicin+cytarabine for } 5 \\
\text { rounds Mitoxantrone+high-dose cytarabine }\end{array}$ & $\begin{array}{l}\text { Relapsed } \\
\text { Refractory }\end{array}$ & 12 months \\
\hline NO. 5 & male & 72 & $\begin{array}{l}\text { AML with } \mathrm{t}(8 ; 21)(\mathrm{q} 22 ; \mathrm{q} 22) \text {; favorable } \\
\text { group with good karyotype }\end{array}$ & $\begin{array}{l}\text { Low-dose cytarabine }+ \text { aclarubicin }+\mathrm{G}-\mathrm{CSF} \\
\text { Idarubicin }+ \text { cytarabine }\end{array}$ & Refractory & \\
\hline NO. 6 & female & 28 & $\begin{array}{l}\text { AML with maturation; Intermediate group } \\
\text { with normal karyotype }\end{array}$ & Idarubicin + cytarabine Idarubicin + cytarabine & Refractory & \\
\hline NO. 8 & female & 39 & $\begin{array}{l}\text { Acute monoblastic /monocytic leukemia; } \\
\text { Intermediate group with }+8\end{array}$ & $\begin{array}{l}\text { Daunarubicin }+ \text { cytarabine for } 4 \text { rounds Decitabine }+ \\
\text { Low-dose cytarabine }+ \text { Homoharringtonine }+\mathrm{G}-\mathrm{CSF} \\
\text { Idarubicin }+ \text { cytarabine for } 2 \text { rounds }\end{array}$ & $\begin{array}{l}\text { Relapsed } \\
\text { Refractory }\end{array}$ & 18 months \\
\hline NO. 9 & male & 56 & $\begin{array}{l}\text { Acute myelomonocytic leukemia; } \\
\text { Intermediate group with normal karyotype }\end{array}$ & $\begin{array}{l}\text { Cladribine+Mitoxantrone+cytarabine } \\
\text { Decitabine+venetoclax } \\
\text { Decitabine+homoharringtonine }+ \text { cytarabine }\end{array}$ & Refractory & \\
\hline NO. 10 & male & 19 & $\begin{array}{l}\text { Acute monoblastic/monocyti c leukemia; } \\
\text { Intermediate group with normal karyotype }\end{array}$ & $\begin{array}{l}\text { Daunarubicin+cytarabine for } 2 \text { rounds Fludarabine+ } \\
\text { high-dose cytarabine + G-CSF Mitoxantrone+high- } \\
\text { dose cytarabine Decitabine+ Low-dose cytarabine + } \\
\text { cladarubine + G- CSF HLA identical stem-cell } \\
\text { transplantation Decitabine + Low-dose cytarabine + } \\
\text { donor lymphocyte infusion }\end{array}$ & $\begin{array}{l}\text { Relapsed } \\
\text { Refractory } \\
\text { Relapsed } \\
\text { Refractory }\end{array}$ & $\begin{array}{l}2 \text { months } \\
3 \text { months }\end{array}$ \\
\hline NO. 11 & male & 68 & $\begin{array}{l}\text { AML without maturation; adverse group } \\
\text { with normal karyotype but ASXL1 mutation }\end{array}$ & none & $\begin{array}{l}\text { Not } \\
\text { available }\end{array}$ & \\
\hline NO. 12 & male & 82 & $\begin{array}{l}\text { AML without maturation; adverse group } \\
\text { with normal karyotype but ASXL1 mutation }\end{array}$ & none & $\begin{array}{l}\text { Not } \\
\text { available }\end{array}$ & \\
\hline NO. 13 & male & 64 & $\begin{array}{l}\text { Acute myelomonocytic leukemia; Adverse } \\
\text { group with complex karyotype including - } \\
7,+8,+8\end{array}$ & none & $\begin{array}{l}\text { Not } \\
\text { available }\end{array}$ & \\
\hline
\end{tabular}

\subsection{Treatment Responses}

Five of the 13 patients achieved CR after the administration of sorafenib with/without low-dose cytorabine. All of them have their bone marrow blast cells decreased to less than 10 percent in 6 weeks (see figure 1). The median time required for inducing $\mathrm{CR}$ is 57 days in the five patients. Their events free survive duration with sorafenib maintenance treatment ranged from 3 to 25 months. Of the 13 patients, 5 received sorafenib and low-dose cytorabine for induction and the remaining 8 patients received sorafenib alone. When comparing their CR after sorafenib treatments, the patients receiving sorafenib and cytorabine had higher $\mathrm{CR}$ rates than the patients given sorafenib alone ( $80 \%$ vs $12.5 \%$ respectively, $\mathrm{P}=.03$ ). 


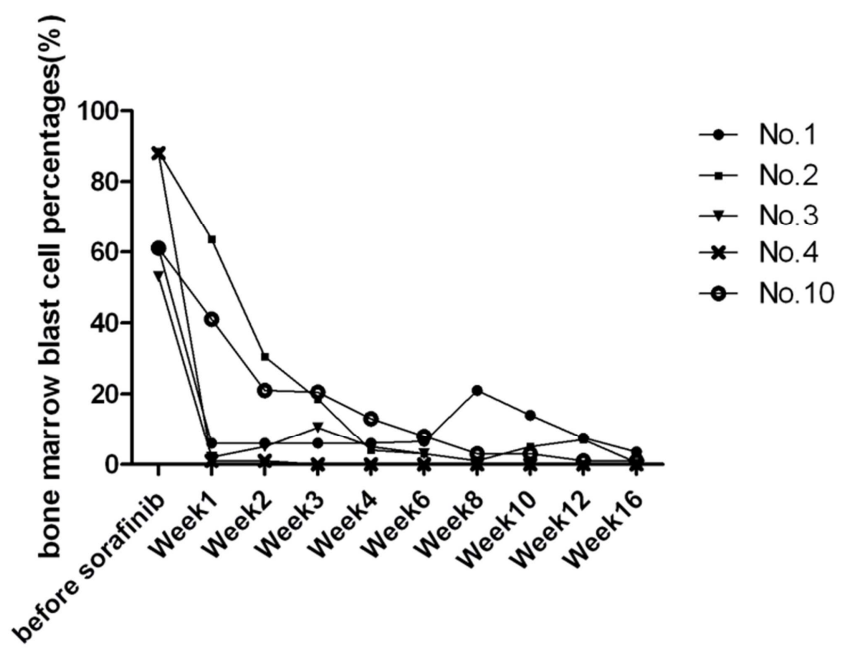

Figure 1. Chronological changes of CR patients' bone marrow blast cell percent.

Four of other 8 refractory patients could also have a temporary decrease of bone marrow blast cell percent in the first week, but the trend didn't sustained in the next two to three weeks. Two other refractory patients showed no response of bone marrow blast cell percent and two had even higher percentages of blast cells after one week of sorafenib usage (see figure 2).

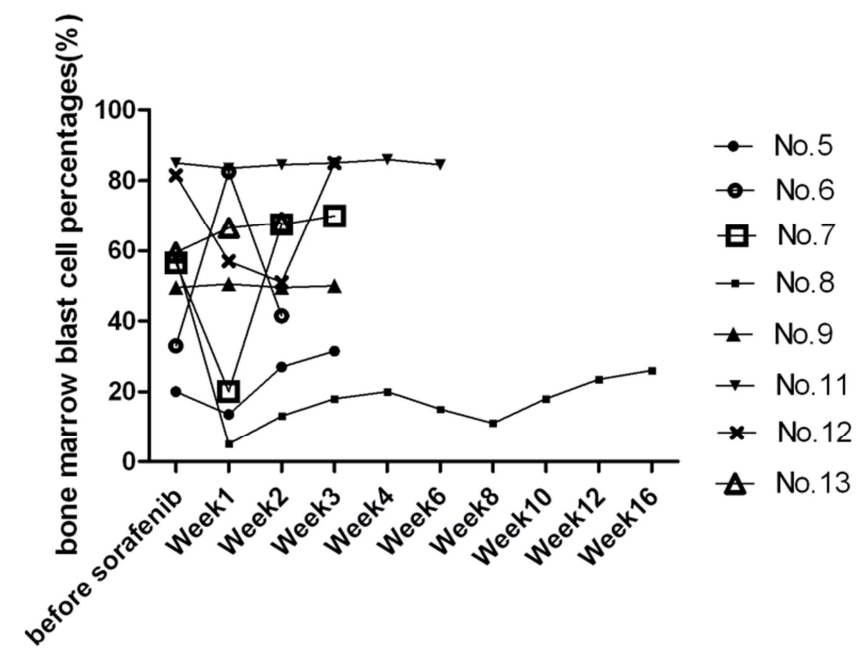

Figure 2. Chronological changes of sorafenib refractory patients' bone marrow blast cell percent.

\subsection{Adverse Events}

All patients have their liver and kidney functions monitored in two weeks after taking sorafinib. Only one patient had mild elevation of her Alanine transaminase at first week, and can be classified into Grade 1 adverse event. Other patients were not found to have any impairment of liver and kidney functions. Only one patient developed palmar-plantar erythrodysesthesia at the day 54 since sorafenib usage. The grade of this adverse event reached to 3 in a week with skin peeling and pain which impacting daily life, and treatments with keratolytics or emollients, pyridoxine and celecoxib administrations were inefficient. The patient had to stop sorafenib temporarily and his syndromes tapered in 1 week. But on re-administration of sorafenib with half planned dose, his skin adverse effect reappeared. Other two patients reported dermal lesion during the usage of sorafenib but their symptoms can be eliminated by cut down the dosage of sorafenib or temporary stop using the drug. Patients didn't report other symptoms which may relate with sorafenib usage and their electrolytes were kept barely normal. During the induction period with sorafenib, all patients achieved CR experienced neutropenia of more than 7 days without fatal systemic infections.

\subsection{Follow-up}

One patient achieved CR with sorafenib received hyperidentical hematopoietic stem cell transplantation 3 months later. Two patients with CR responses stopped taking sorafenib for side effect at day 69 or having kept in CR for 12 months. They dyed of relapses of leukemia at 2 months and 9 months later. Two other patients with CR stopped taking sorafenib as maintenance therapy for later relapses at the $11^{\text {th }}$ and the third month respectively. They retrieved to salvage chemotherapy but failed and dyed of neutropenic infection or intracranial bleeding. The median follow-up duration for the whole cohort has been more than 11 months. The median survival time of the 5 patients achieved CR with sorafenib was 520 days, while the median survival time of the 8 patients without CR with sorafenib was 344 days. We found no significant survival differences between the patients achieved CR with sorafenib and those patients without CR (see figure 3).



+ achieved CR

- +. without $\mathrm{CR}$

Figure 3. Kaplan-Meier curve of sorafenib induced CR or resistant patients.

\section{Discussion}

Our present observation showed the encouraging efficiency of $\mathrm{CR}$ induction with sorafenib in $\mathrm{R} / \mathrm{R}$ AML or geratic patients without FLT3-ITD mutation. Especially in combination with low-dose cytorabine, sorafenib induced 4 of 5 such formidable patients to $\mathrm{CR}$, which is significantly more efficient than sorafenib treatment alone.

The CR rate is at least not much inferior to the $\mathrm{CR}$ rates of former combining chemotherapies. In updated NCCN guidelines for AML, to make salvage of $\mathrm{R} / \mathrm{R}$ AML patients, aggressive regimens, such as cladribine, cytarabine and granulocyte colony-stimulating factors with/without idarubicin or high-dose cytarabine with anthracyclines, are intended for those can tolerate such therapies. But the results 
are not satisfying. For example, in a recent large sample analysis, the combination of fludarabine, high-dose cytarabine, and granulocyte clony-stimulation factor (FLAG) plus idarubine and gemtuzumab-ozogamicin induced CR/CRi of $51 \%$ with $9 \%$ induction death in salvage of $\mathrm{R} / \mathrm{R}$ AML patients [15]. Meanwhile, a recently reported outcome of FLAG regimen for R/R AML gave the CR/CRi rate of $41.7 \%$ with regimen associated mortality of $37.5 \%$, and the mortality was even higher for those used FLAG plus idarubine [16]. The result of FLAG regimen plus other anthracyclines is still under observation. For example, FLAG plus mitoxantrone was reported to result in $59 \% \mathrm{CR}$ in $\mathrm{R} / \mathrm{R}$ AML patients with $14 \%$ early death rate [17]. But the same regimen yielded $73 \% \mathrm{CR}$ with $2.4 \%$ early death rate in a recent report which may be related with lower number of adverse karyotype and younger median age [18]. Other highdose cytarabine included regimens such as HAMA (comprised of high-dose cytarabine, mitoxantrane and Lasparanginase) or very high dose single-agent cytarabine resulted CR of $41 \%$ to $60 \%$ and early mortality of $4 \%$ to $18 \%$ in induction $[19,20]$. The addition of clofarabine, a new generation deoxyadenosine analog, to high-dose cytarabine was reported to induce the overall response rate of only $21 \%$ and the 30 -day mortality was as high as $21 \%$ [4]. Another deoxynuleoside analog, cladribine, in combination with highdose cytarabine was reported to yield $57.9 \% \mathrm{CR}$ in $\mathrm{R} / \mathrm{R}$ AML patients. And those of age above 60 were still related with an unfavourable CR [21]. So the aggressive salvage chemotherapies usually associated with compromising results of $\mathrm{CR}$ induction at the expense of considerable induction mortalities. Our sorafenib treatment yielded an acceptable $\mathrm{CR}$ rate especially in combination with low-dose cytarabine, and no early death happened during induction. Further the side effects were mostly mild and can be alleviated quickly by drug reduction or cessation. Our CR rate even seems better than that of other latterly published phase II trials on $\mathrm{R} / \mathrm{R}$ AML, in which novel regimens only resulted overall response rates of 14 to $33 \%$ [22, 23].

As to the elderly AML patients unfit for intensive chemotherapy, single low-dose cytorabine demonstrated modest $\mathrm{CR}$ rate of $18 \%$ leading to improved survival by comparing with best supportive care [9]. Our sorafenib treatment in 3 old patients unfit for convenient chemotherapies showed no successful CR induction, but it temporarily decreased bone marrow blast cell percentage in 1 of the 3 old unfit patients and successfully induced CR in two R/R AML patients aged more than 70 (patient No. 3 and 4) in combination with low dose cytarabine. Given the small sample of old patient treatment with sorafenib, we still need further observation of sorafenib efficiencies in the old patients especially for those unfit to intensive chemotherapies.

Our patients are all FLT3-ITD mutation negative, which is pivotally insensitive to sorafenib. But in present observation, single sorafenib did temporarily decrease the bone marrow blast cells and suppress patients' white cells increment, spare time to refresh post intensive chemotherapy, and even induce
$\mathrm{CR}$ in one patient. One recent article also reported cases that single sorafenib could yield shrink of extramedullary myeloid tumour in one young refractory AML and improvement of hematologic parameters in one old relapsed AML. All of the patients were FLT3-ITD mutation negative [24]. The results suggested single sorafenib treatment could do some good to $\mathrm{R} / \mathrm{R}$ AML patients without FLT3-ITD mutation and we may improve antileukemic effect by adding sorafenib with other antitumor drugs.

Because sorafenib is a multi-targets tyrosine kinase inhibitor including Raf/MEK/ERK pathway and receptor tyrosine kinases such as vascular-endothelial growth factor receptor 2, platelet derived growth factor receptor $\beta$, FMS like tyrosine kinase 3 and CD117. It may synergistically act with cytotoxic drug to increase the treatment responses of leukemia. Our present analysis of sorafenib treatment responses supported that the combination of sorafenib with cytarabine was more efficient in $\mathrm{CR}$ induction than single sorafenib. It is consistent with previous in vitro study that sorafenib could increase the cellular accumulation of cytarabine and its metabolites resulting in addictive to synergistic antileukemic activity [25].

Our results also supported sorafenib treatment to act as a bridge for transplantation in tough handling leukaemia patients. For example, one primary chemotherapy resistant AML patient in our study received hyper-identical hematopoietic stem cell transplantation after achieving CR with sorafenib and has survived with event free for almost 3 years by now. But two other R/R AML patients achieved CR by sorafenib relapsed within 11 months and still failed of further salvage chemotherapies. The results suggested the sorafenib maintenance treatment couldn't long time suppress leukemia and further consolidation therapy or other cure method should be planned on time.

As a primary study on sorafenib treatment of AML patients without FLT3-ITD mutation, there are obvious shortcomings in this report. First, the relationship of c-Kit mutation and sorafenib responses in our $\mathrm{R} / \mathrm{R}$ patients still needs further study, because c-Kit mutation also related with fail of chemotherapy and its downstream signals, such as Ras/ERK and PI3K, can be considerably affected by sorafenib. The impact of sorafenib on the $\mathrm{R} / \mathrm{R}$ or geratic AML may related with $\mathrm{c}-\mathrm{Kit}$ mutation in these patients. Second, the limited sample size refrained us from stratifying the patients by subgroups of R/R AML or old AML unfit for chemotherapy. So we can not statistically verify the advantage of combining sorafenib with cytarabine in separate subgroups by now.

\section{Conclusion}

Induction with sorafenib especially in combination with low-dose cytarabine showed considerable $\mathrm{CR}$ rate, rare induction mortality and acceptable survival duration in our small sample of AML patients without FLT3-ITD mutation. Further observations of the regimen and studies of underlying mechanisms deserves to be made.

Acknowledgements: we are particularly grateful to 
Guangdong science and technology department for its generous support to this research by fund of Science and Technology Planning Project of Guangdong Province (No. 2014A020212664) and fund of special Project of Science and Technology in Guangdong Province (No. 2017B020247011).

\section{References}

[1] Walter RB, Kantarjian HM, Huang X, et al. Effect of complete remission and responses less than complete remission on survival in acute myeloid leukemia: a combined Eastern Cooperative Oncology Group, Southwest Oncology Group, and M. D. Anderson Cancer Center Study. J Clin Oncol. 2010; 28 (10): 1766-71.

[2] Yanada M, Garcia-Manero G, Borthakur G, et al. Potential cure of acute myeloid leukemia: analysis of 1069 consecutive patients in first complete remission, Cancer. 2007; 110 (12): $2756-2760$.

[3] Breems DA, Van Putten WL, Huijgens PC, et al. Prognostic index for adult patients with acute myeloid leukemia in first relapse. J Clin Oncol. 2005; 23 (9): 1969-78.

[4] Roberts DA, Wadleigh M, McDonnell AM, DeAngelo DJ, Stone RM, Steensma DP. Low efficacy and high mortality associated with clofarabine treatment of relapsed/refractory acute myeloid leukemia and myelodysplastic syndromes. Leuk Res. 2015; 39 (2): 204-10.

[5] Faderl S, Wetzler M, Rizzieri D, et al. Clofarabine plus cytarabine compared with cytarabine alone in older patients with relapsed or refractory acute myelogenous leukemia: results from the CLASSIC I Trial. J Clin Oncol. 2012; 30 (20): 2492-9.

[6] Juliusson G, Antunovic P, Derolf A, et al. Age and acute myeloid leukemia: real world data on decision to treat and outcomes from the Swedish Acute Leukemia Registry. Blood. 2009; 113 (18): 4179-87.

[7] Appelbaum FR, Gundacker H, Head DR, et al. Age and acute myeloid leukemia. Blood. 2006; 107 (9): 3481-5.

[8] Röllig C, Thiede C, Gramatzki M, et al. A novel prognostic model in elderly patients with acute myeloid leukemia: results of 909 patients entered into the prospective AML96 trial. Blood. 2010; 116 (6): 971-8.

[9] Burnett AK, Milligan D, Prentice AG, et al. A comparison of low-dose cytarabine and hydroxyurea with or without all-trans retinoic acid for acute myeloid leukemia and high-risk myelodysplastic syndrome in patients not considered fit for intensive treatment. Cancer. 2007; 109 (6): 1114-24.

[10] Röllig C, Serve H, Hüttmann A, et al. Addition of sorafenib versus placebo to standard therapy in patients aged 60 years or younger with newly diagnosed acute myeloid leukaemia (SORAML): a multicentre, phase 2, randomised controlled trial. Lancet Oncol. 2015; 16 (16): 1691-9.

[11] Ravandi F, Alattar ML, Grunwald MR, et al. Phase 2 study of azacytidine plus sorafenib in patients with acute myeloid leukemia and FLT-3 internal tandem duplication mutation. Blood. 2013; 121 (23): 4655-62.
[12] Patel JP, Gönen M, Figueroa ME, et al. Prognostic relevance of integrated genetic profiling in acute myeloid leukemia. $\mathrm{N}$ Engl J Med. 2012; 366 (12): 1079-89.

[13] Chevallier P, Labopin M, Turlure P, et al. A new Leukemia Prognostic Scoring System for refractory/relapsed adult acute myelogeneous leukaemia patients: a GOELAMS study. Leukemia. 2011; 25 (6): 939-44.

[14] Webster JA, Pratz KW. Acute myeloid leukemia in the elderly: therapeutic options and choice. Leuk Lymphoma. 2018; 59 (2): 274-287.

[15] Bergua JM, Montesinos P, Martinez-Cuadrón D, et al. A prognostic model for survival after salvage treatment with FLAG-Ida +/- gemtuzumab-ozogamicine in adult patients with refractory/relapsed acute myeloid leukaemia. $\mathrm{Br} \mathrm{J}$ Haematol. 2016; 174 (5): 700-10.

[16] Farooq MU (1), Mushtaq F (1), Farooq A (2), et al. FLAG vs FLAG-IDA: outcomes in relapsed/refractory acute leukemias. Cancer Chemother Pharmacol. 2019; 83: 1191-1193.

[17] Hänel M, Friedrichsen K, Hänel A, et al. Mito-flag as salvage therapy for relapsed and refractory acute myeloid leukemia. Onkologie. 2001; 24: 356-60.

[18] Hatsumi N, Miyawaki S, Yamauchi T, et al. Phase II study of FLAGM (fludarabine + high-dose cytarabine + granulocyte colony-stimulating factor + mitoxantrone) for relapsed or refractory acute myeloid leukemia. Int J Hematol. 2019; 109: 418-425.

[19] Ahmed T, Holwerda S, Klepin HD, et al. High dose cytarabine, mitoxantrone and l-asparaginase (HAMA) salvage for relapsed or refractory acute myeloid leukemia (AML) in the elderly. Leuk Res. 2015; 39 (9): 945-9.

[20] Wolach O, Itchaki G, Bar-Natan M, et al. High-dose cytarabine as salvage therapy for relapsed or refractory acute myeloid leukemia--is more better or more of the same? Hematol Oncol. 2016; 34 (1): 28-35.

[21] Wang $\mathrm{L}, \mathrm{Xu} \mathrm{J}$, Tian X, et al. Analysis of Efficacy and Prognostic Factors of CLAG Treatment in Chinese Patients with Refractory or Relapsed Acute Myeloid Leukemia. Acta Haematol. 2019; 141: 43-53.

[22] Litzow MR, Wang XV, Carroll MP, et al. A randomized trial of three novel regimens for recurrent acute myeloid leukemia demonstrates the continuing challenge of treating this difficult disease. Am J Hematol. 2019; 94: 111-117.

[23] Daver N (1), Garcia-Manero G (2), Basu S (3), et al. Efficacy, Safety, and Biomarkers of Response to Azacitidine and Nivolumab in Relapsed/Refractory Acute Myeloid Leukemia: A Nonrandomized, Open-Label, Phase II Study. Cancer Discov. 2019; 9: 370-383.

[24] Yang N, Gu Z, Liu Z, et al. Sorafenib as a Salvage Therapy in FLT3-ITD Negative Relapse/ Refractory Acute Myeloid Leukemia. Anticancer Agents Med Chem. 2018; 18: 14891494.

[25] $\mathrm{Hu} \mathrm{S}$, Niu $\mathrm{H}$, Inaba $\mathrm{H}$, Orwick $\mathrm{S}$, et al. Activity of the multikinase inhibitor sorafenib in combination with cytarabine in acute myeloid leukemia. J Natl Cancer Inst. 2011; 103 (11): 893-905. 